\title{
Flexible decision-strategy improvements in children
}

\author{
Nicolas W. Schuck ${ }^{1 *}$, Amy X. Li ${ }^{2}$, Dorit Wenke ${ }^{3}$, Destina S. Ay ${ }^{4}$, Anika Löwe $^{1}$, \\ Robert Gaschler ${ }^{5} \&$ Yee Lee Shing 6,7
}

${ }^{1}$ Max Planck Research Group NeuroCode, Max Planck Institute for Human Development, Berlin

2 School of Psychology, University of New South Wales, Sydney

${ }^{3}$ PFH Private University of Applied Sciences Göttingen

4 Department of Psychology, Universität Potsdam

${ }^{5}$ School of Psychology, FernUniversität Hagen

${ }^{6}$ Institute of Psychology, Goethe University Frankfurt

${ }^{7}$ Department of Lifespan Psychology, Max Planck Institute for Human Development, Berlin

Word Count: 7052 words

Character Count: 38001 characters (not including spaces)

${ }^{*}$ Corresponding author contact:

Max Planck Research Group NeuroCode

Max Planck Institute for Human Development

Lentzeallee 94, 14195 Berlin, Germany

schuck@mpib-berlin.mpg . de

tel: +493082406649 


\begin{abstract}
Children often perform worse than adults on tasks that require focused attention. While this is commonly regarded as a sign of incomplete cognitive development, a broader attentional focus could also endow children with the ability to find novel solutions to a given task. To test this idea, we investigated children's ability to discover and use novel aspects of the environment that allowed them to improve their decision-making strategy. Participants were given a simple choice task in which the possibility of strategy improvement was neither mentioned by instructions nor encouraged by explicit error feedback. In two experiments, 39 adults executed the instructed strategy well, but only $28.2 \%$ of participants improved their task strategy with time. Children $(\mathrm{n}=$ 47, 8 - 10 years of age) made approximately twice as many errors in executing the instructed choice rule, but were as likely as adults to improve their strategy $(27.5 \%$ of participants). A task difficulty manipulation did not affect results. The lack of age differences in flexible strategy updating was contrasted not only by substantial differences in task-execution, but also by reduced working memory and inhibitory control in children relative to adults. Our results suggest that children have adult-level abilities to find alternative task solutions. This capacity does not depend on adult-level cognitive control.
\end{abstract}

\title{
Highlights
}

- Children show adult-level abilities to discover and employ alternative strategies without instructions

- Instructed task performance, working memory, and response inhibition are less functional compared to adults

- Results are replicated across two experiments 
25

\section{Introduction}

Humans develop into remarkably adaptive and efficient decision makers over the first two decades of their lives. Of particular importance for this process is the development of cognitive control functions, which allow us to keep information about the ongoing task in working memory and shield it from interference by irrelevant distractions (Corbetta \& Shulman, 2002; Sakai, 2008). Developmental research has therefore often focused on children's improvements in these functions (e.g. Baum et al., 2017; Bunge \& Zelazo, 2006, Bunge \& Wright, 2007; Feo, Panzeri, \& Dehaene, 2018; Gur et al., 2012).

Yet, truly flexible goal-directed behavior also involves improving one's current decision making strategy. Similar to how discovering unknown connections can allow shortcuts in navigation, learning about previously ignored or novel contingencies in the environment can lead to behavioral or cognitive changes that achieve the same goal in a more efficient manner. The disadvantage that children have with executing some tasks could hence lead to a somewhat paradoxical advantage for finding better strategies; "shortcuts" which can only be discovered by processing information that is irrelevant for the current strategy. In line with this idea, children have been found to outperform adults in detecting changes in shapes they were not cued to attend and in remembering information that is irrelevant for the instructed task (Plebanek \& Sloutsky, 2017). This suggests that children may tend to distribute attention across multiple aspects of stimuli, including those that are not relevant to the instructed goal. Other research has also suggested that children may be more eager to explore less known options than adults (Schulz, Wu, Ruggeri, \& Meder, 2019), or to sample hypotheses in a more probabilistic fashion (Bonawitz, Denison, Griffiths, \& Gopnik, 2014). Likewise, a number of previous findings have shown that children are remarkably variable in the strategies they employ, when even performing the same task (Siegler, 1995 . Gaschler, Vaterrodt, Frensch, Eichler, \& Haider, 2013) and emphasized that children usually use a variety of approaches to problem solve (Siegler, 1996, 1997, 2006). Frequent task switching, in turn, is known to weaken task maintenance or 'shielding' (Dreisbach \& Wenke, 2011). In combination, these characteristics might allow children to be surprisingly good in 
adaptive strategy updating, which is generally regarded as a complex computational problem (Marewski \& Link, 2014; Lieder \& Griffiths, 2017).

This idea stands in contrast to a large developmental literature that has shown that efficient decision making is comparatively slow to mature fully (Diamond, 2013, Gur et al., 2012). Compared to the development of other cognitive faculties, such as language or motor skills, decision making that involves multiple features becomes mature particularly late in development, and reaches adult-levels only in late adolescence (Davidson, Amso, Anderson, \& Diamond, 2006 Garon, Bryson, \& Smith, 2008). Likewise, the ability to focus attention on task-relevant aspects and to suppress distracting information has been found to be less effective in children in a variety of tasks, such as the anti-saccade (Fischer, Biscaldi, \& Gezeck, 1997; Fukushima, Hatta, \& Fukushima, 2000), Flanker (Ridderinkhof, Van Der Molen, Band, \& Bashore, 1997) or Stroop (Tipper, Bourque, Anderson, \& Brehaut, 1989) tasks and working memory capacity also does not reach adult levels until late adolescence (Demetriou, Christou, Spanoudis, \& Platsidou, 2002). Interestingly, even the ability to follow explicit rules continues to enhance as children become older in middle childhood, thereby contributing to the protracted development of children's control of behavior (Bunge \& Zelazo, 2006). Over the same period of time, children become increasingly able to integrate and execute different rules according to the cues provided by task context (Bunge \& Wright, 2007), particularly starting from late childhood on (Davidson et al., 2006). Finally, modelbased decision making is also known to develop slowly (Decker, Otto, Daw, \& Hartley, 2016). Neuroscientific research that has linked the protracted cognitive development to the relatively delayed maturation of the prefrontal cortex (e.g. Hartley \& Somerville, 2015 Blakemore \& Choudhury, 2006). Studies focusing on structural brain development have for instance found links between change in cortical thickness in the anterior cingulate cortex and cognitive flexibility (Kharitonova, Martin, Gabrieli, \& Sheridan, 2013), and different aspects of cognitive flexibility have been linked to different subregions of the prefrontal cortex (Bunge \& Zelazo, 2006). In addition, studies of functional brain development have shown that brain activation patterns and long-range connectivity involved in cognitive control continue to 
change throughout childhood (Luna, Padmanabhan, \& O'Hearn, 2010).

The research summarized above suggests that cognitive control skills, and their underlying neural processes, mature slowly. Considerably less is known, however, what impact these weaknesses have on the ability of children to flexibly update decision-making strategies. The main goal of the present paper is therefore to ask how good children are in discovering and updating an ongoing decision-making process with an alternative strategy that achieves the same goal. This aspect of cognitive flexibility lies not in being able to identify the relevant rule based on the context. Rather it relies on the ability to assess the potential usefulness of seemingly unimportant information in the environment that may afford the discovery of a new strategy (strategy exploration). As we noted above, the relative weakness of task 'shielding' (Dreisbach \& Haider, 2008) seen in children could potentially turn out to be beneficial for their ability to discover alternative strategies. In addition, children are not as influenced by instructions as adults are (Decker, Lourenco, Doll, \& Hartley, 2015), but are comparatively sensitive to statistical regularities in their environment that are important for language learning (Evans, Saffran, \& Robe-Torres, 2009; Saffran, Aslin, \& Newport, 1996). One might therefore expect that children, due to their lower ability to inhibit irrelevant information and to follow instructions as well as their sensitivity to statistical regularities, may have an advantage or at least an equal level of alternative strategy discovery abilities compared to young adults.

\section{Experiment 1}

Experiment 1 tested children and young adults with the Spontaneous Strategy Switch Task, which assesses the ability to discover and implement a novel strategy (Schuck et al., 2015, Gaschler, Schuck, Reverberi, Frensch, \& Wenke, 2019). Participants were instructed to perform a simple decision making task that required responding to the spatial location of a stimulus with two different buttons. Unbeknownst to participants, the stimulus color was fully correlated with the required response, such that participants in principle could use an alternative strategy and respond to stimulus color rather than stimulus location. 
Our previous work has shown that about one third of adult participants will discover and use the alternative strategy. The same data also indicated that strategy switches occurred abruptly (within a few minutes) and occurred throughout the experiment. In Experiment 1 we asked how frequent strategy discovery is among children compared to adults, and if the characteristics of strategy change differ between age groups.

\section{Methods}

\section{Participants}

Twenty-eight children and 22 young adults were tested in Experiment 1. Participants were excluded if they failed to perform the instructed color task, as tested by a binomial test assessing performance against chance in the final two blocks (blocks 9 and 10) ( $\alpha=5 \%$ ). This led to the exclusion of 6 children and 1 adult. The effective sample size therefore consisted of 22 children (11 female) with a mean age of 9.5 years $(\mathrm{SD}=2.5$, range $=20$ to 30 years) and 21 young adults (8 female) with a mean age of 22.7 years $(\mathrm{SD}=0.8$, range $=8$ to 10 years). All participants provided informed consent and all applicable ethical regulations related to research with human participants were followed. The ethics board of the Max Planck Institute for Human Development approved all reported studies.

\section{Main task}

Stimuli Each stimulus consisted of 72 small colored squares that were distributed uniformly over a rectangular patch $(120 \times 120 \mathrm{px})$, covering half of the patch area. The patch of colored squares was displayed within a grey reference frame that was slightly larger than the patch $(150 \times 150 \mathrm{px})$. The patch itself was presented centrally on the screen, but on each trial the reference frame was offset from the center by \pm 5 px on the horizontal and vertical axes, see Fig. 1A. The patch was therefore closer to one of the four corners of the reference frame. Offsets changed trial-wise and participants were instructed to decide where the patch was positioned within the frame, i.e to which of the four corners of the reference frame it was closest to. To respond, participants used two response keys ([x] and [,] marked with a 
white label on a QWERTZ keyboard). One key had to be pressed whenever the stimulus was closer to the upper left or the lower right corner of the frame, whereas the other key was correct for the opposed corners (lower left and upper right). The response to corner mapping was randomized across participants.

On each trial, the squares that made up the patch had the same color and were either green or red. Participants were not informed that the colors had any meaning for the task and the colors indeed changed randomly during the first block (50\% red and 50\% green patches for each response button). After this block, however, the stimulus color was consistently paired with the required response button (Fig. 1B). This meant that in trials requiring a left response (upper left or lower right corner), the patch was for instance always green, whereas in trials requiring a right response the patch was always red. If this was noticed by a participant, it allowed her to change her decision-making strategy from selecting buttons based on patch location to responding based on patch color. The color-button mapping was counterbalanced across participants.

Trial types The main task included four different trial types that involved slightly different response requirements (Fig 1C). In standard trials, the patch and the reference frame appeared simultaneously for $400 \mathrm{~ms}$ on the screen and participants could respond as instructed immediately after stimulus onset. In LateGo trials, the patch appeared for 2000 ms before the reference frame appeared for $400 \mathrm{~ms}$ in addition to the patch. Participants were instructed to withhold responding until the frame was displayed. NoGo trials were identical to LateGo trials, except that the frame did not appear after $2000 \mathrm{~ms}$ and the task continued with the next trial. Participants needed to withhold responding in these trials. Finally, in ambiguous trials, the frame appeared simultaneously with the colored patch, but was not offset from the center. Hence the patch was not closer to any of the four corners and responding based on relative spatial position of the patch would lead to random choice behavior in ambiguous trials. 
A

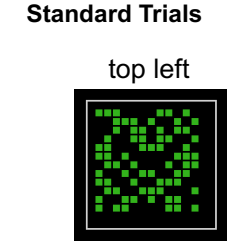

Responses: $\bigcirc \bigcirc$

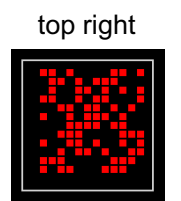

$\bigcirc \bigcirc$

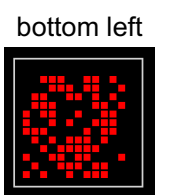

$\bigcirc \bigcirc$

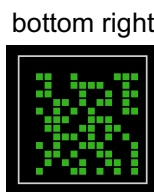

$\bigcirc \bigcirc$

B Block (each 180 trials)

Experiment 1

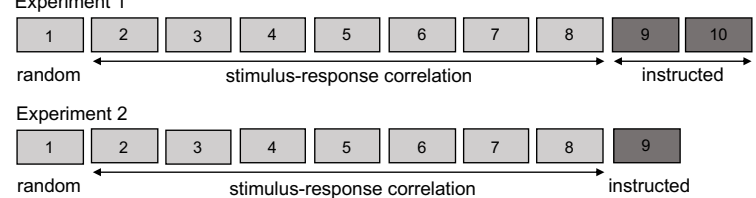

C
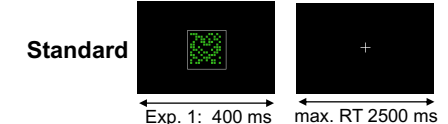

$\stackrel{\leftrightarrow}{\stackrel{m a x}{R} \text { RT } 2500 \mathrm{~ms}}$
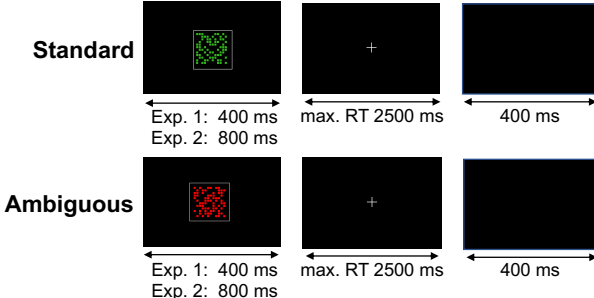

LateGo
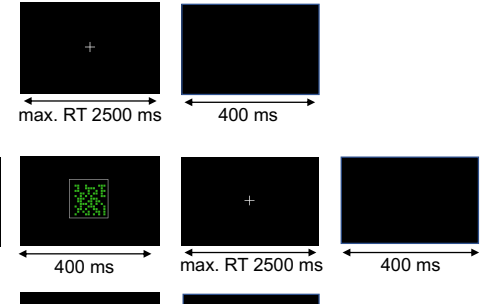

NoGo

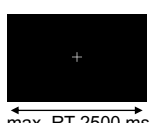

$400 \mathrm{~ms}$

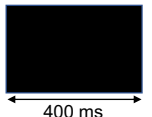

Figure 1: Stimulus and Task Design. (A): Stimulus response mapping in standard trials. The mapping was counterbalanced across participants. Each trial involved one patch of colored squares inside a light reference frame as shown. The colored squares were shifted systematically from the center of the frame and participants had to decide which corner of the white frame the patch is closer to. (B): Block order for Experiments 1 and 2. Each block started with a block in which stimulus color and corner were uncorrelated ("random blocks"). Without notifying participants, from block 2 on the required response and the stimulus color had a fixed relation in all standard trials. After block 8, participants were instructed to use the color to determine their response ("instructed blocks"). Experiments 1 and 2 differed regarding the number of instructed blocks. (C): Trial structure for standard, ambiguous, LateGo and NoGo trials. Each row shows the onset and duration of the colored squares, the white frame, the fixation cross and the response stimulus interval for one condition, see labels.

\section{Additional measures}

Questionnaire Following the main experiment, participants were asked to fill out a questionnaire containing several questions about the task. These questions asked (1) whether the hidden color rule was noticed [yes/no], (1b) if yes, when within the experiment it was noticed [participants indicated the proportion of elapsed time before noticing on a clockface], (2) whether the discovered color rule was used to make decisions [yes/no], (3) to report the rule by writing down which color was associated with which corner. Due to human error, questionnaire data from one adult participant were lost. Only analyses which considered the questionnaire data were constrained to include participants for which task and questionnaire data was available. 
Working memory test Participants completed a digit-sorting task as a measure of working memory. For each trial, a set of numbers was verbally read out by the experimenter. After the last number was presented, participants were asked to write down the numbers in the ascending order on the answer sheet. A total of 15 sets of numbers divided into five levels were used, starting from four numbers at the first level and one number was added for each consecutive level. A set of numbers was assessed as incorrect if a number was missing or if the sequence was not in the correct order. A maximum of fifteen points could be scored on the task. Due to technical errors, WM data from 6 participants were lost (3 younger adults). Only analyses which considered the WM performance were constrained to include participants for which task and WM data was available.

Stroop Test A Stroop task was used as a measure of inhibition. The task consisted of 40 congruent, 40 incongruent, and 40 neutral trials. Participants were instructed to respond according to the font color of the stimulus word (e.g., for words shown in blue color, press the blue key). For congruent trials, the stimulus words ("BLUE" or "YELLOW") in their corresponding colors were presented on the screen. For incongruent trials, the stimulus words were shown with non-corresponding colors. For neutral trials, the stimulus word was "XXX" and was either shown in blue or yellow color. We computed two scores: the difference between reaction times in neutral and in congruent trials (semantic facilitation), and the difference between neutral and incongruent trials, the so called semantic interference score. Due to human and technical errors, Stroop data from seven participants was lost (six younger adults and one child, same participants for which working memory was lost plus participants for which erroneously the wrong computer program was used). The task was implemented in ePrime. Due to technical errors, Stroop data from 2 participants were lost. Analyses which considered Stroop performance therefore excluded two additional participants.

\section{Procedures}

The experiment began with instructions for the main task. Participants were explained that, on each trial, they should make a response based on the spatial position of the 
patch within the reference frame. While children received instructions verbally to ensure correct understanding, young adults read the same instructions themselves on the screen. Instructions did neither facilitate nor discourage color use, mentioning only that "each patch will be either red or green". Examples for each corner were shown in both colors. A printout showing the corner-response mapping was attached to the wall in front of the participants, allowing them to refer to it throughout the experiment. Instructions explained all trial types and were followed by a pretraining that ensured that the rules were understood. The pretraining phase lasted for at least 50 trials and was ended once the participant made less than $20 \%$ errors in 24 consecutive trials. Participants received trialwise error feedback on the monitor during this part of the experiment, informing them when the given answer was incorrect, too late, or premature. Colors changed randomly in this part of the task.

After pretraining the main task started and lasted for 10 blocks of 180 trials each in Experiment 1 (Fig.1B). Each block contained 80 standard, 32 ambiguous, 32 NoGo, 16 LateGo trials. In order to discourage counting strategies, 12 additional trials were distributed randomly across trial types such that number of trials per condition varied slightly between blocks. Participants could take a short break after each block. During the main task, no trialwise feedback was given. If the block-wise error rate exceeded $20 \%$, a warning about too many errors was displayed in the break between blocks.

During the first block ("random block"), the color in left and right response trials was chosen at random. From Block 2 on, the color was associated with the correct response as described above. In the break before Block 9, participants were informed that the color and the response were paired. They were not informed about the exact nature of the pairing but rather asked to find the relation and base their responses on the color for the remainder of the experiment ("instructed blocks"). During this break, but before receiving instructions about the color task, participants were also asked to complete a questionnaire assessing knowledge of the color strategy (see above). Then they completed blocks 9 and 10. A subgroup of participants in Experiment 1 erroneously performed an additional 9th block before performing two instructed blocks (4 children and 5 young adults). This did not 
affect the first 8 blocks for those participants and data from this additional block were therefore not analyzed. After the main task and questionnaire were completed, participants performed the Stroop and working memory tasks. The overall duration of the experiment was approximately 160 minutes for children and 120 minutes for young adults.

\section{Analyses}

All analyses were performed using R ( $\mathrm{R}$ Core Team, 2018), employing the 'Ime4' package for mixed effects modelling (Bates, Mächler, Bolker, \& Walker, 2015). Post-hoc tests were adjusted using the Tukey method as implemented in the package 'emmeans'. T-tests were corrected for variance inhomogeneity using the Welch test implemented in R. Unless otherwise noted, mixed effects models included a random intercept and slope of the linear factor Block per subject as well as fixed effects for the factors Block and Age group ('Young Adults' vs. 'Children'). To determine whether participants understood the task, we tested individually whether the percentage of correct regular trials in the last two blocks was significantly different from chance (based on binomial test against chance at $\alpha=.05$ ). This resulted in cut-offs of min. $65 \%$ correct color-based responses, ensuring that only performance of participants was analyzed who had the ability to perform the spatial task in principle.

Switch Point Analysis We used the CUSUM method to determine the block when participants started using the color (as in Schuck et al., 2015). For each participant, we first calculated the average percent of color use over all blocks. We then subtracted this overall mean from each block-wise mean, and calculated the cumulative sum of these differences. Because the differences are negative while the block-wise performance is below the overall mean, and positive once the percent color use is above the mean, the cumulative sum of the differences will decrease until a participant switches and start to increase afterwards. Switch time-points were therefore determined as the time-point at which each participants' cumulative difference score was at its minimum. 


\section{Results}

\section{Instructed task execution}

Errors in blocks 1-8 during regular trials decreased with practice and consistently differed between children and young adults, as reflected in main effects of Block $\chi^{2}(1)=8.6, p<.001$ and Age group, $\chi^{2}(1)=32.3, p<.001$, respectively (see Fig. 2A). Post hoc tests confirmed that the main effect of Age group was driven by younger adults committing less errors than children $(25.3 \%$ vs. $7.7 \%, p<.001)$. This difference persisted throughout the task and remained present in the last two blocks before the color instruction (blocks 7-8), $p<.001$. No interaction between Age group and Block was found. Likewise, reaction times (RTs) differed between age groups, $\left(988 \mathrm{~ms}\right.$ vs $\left.653 \mathrm{~ms}, \chi^{2}(1)=38.3, p<.001\right)$ and decreased with practice, $\chi^{2}(1)=41.5, p s<.001$ (Fig. 2B). Group differences persisted until the last blocks as evidenced by planned comparisons of the average RT in blocks 7 and $8, p<.001$.

Investigating performance during the final instructed block revealed that adults still outperformed children after participants had been provided with instructions to use color: error rates of children and adults were $10.5 \%$ vs. $2.6 \%, p<.001$. In addition, children benefited more from the instructions than adults in terms of error rates, interaction Block (7/8 vs. $9 / 10)$ by Age group, $\chi^{2}(1)=8.5, p<.001$. The same pattern was found concerning RTs, i.e. we found a main effect of Age group in Blocks 9/10 and an interaction between Block $(7 / 8$ vs $9 / 10)$ and Age group, $\chi^{2}(1)>10, p s<.001$.

\section{Response inhibition and working memory}

We next investigated age differences in markers of executive control during task performance and in our control tasks. To characterize response inhibition, we analysed false alarm rates in LateGo and NoGo trials during the main task. This analysis showed that children and adults differed markedly in their response inhibition ability, similarly to the performance disparity seen in standard trials. Specifically, compared to younger adults children made significantly more premature key presses (i.e., responses before the frame was displayed, henceforth "False Alarms") in LateGo trials (12.2\% vs.1.7\%, $\chi^{2}(1)=11.5, p<.001$, Fig $\left.2 \mathrm{C}\right)$ as well as in NoGo 

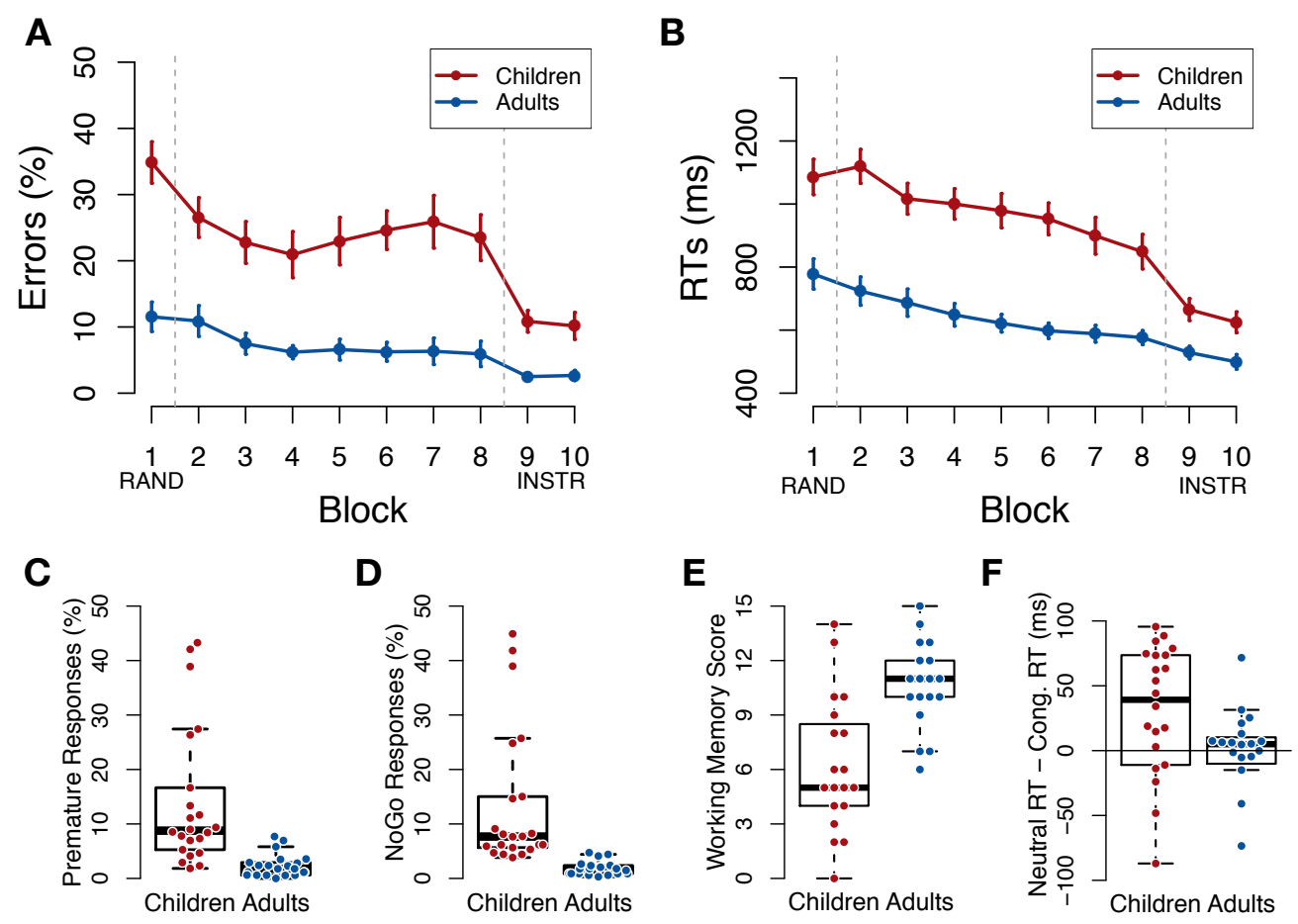

Figure 2: Performance in standard trials and control tasks. (A): Error rates as a function of block separately for children (red) and younger adults (blue) in Experiments 1. As can be seen, large age differences in error rates persisted throughout all blocks. (B): Average reaction times (RTs, in milliseconds) over blocks, also indicating sizable and persistent differences between children and young adults. Colors as in panel A. (C): Percentage of false alarms in LateGo trials among young adults (blue) and children (red) in Experiment 1, indicating significantly less errors among young adults. (D): Percentage of false alarms in NoGo. As in panel (C), younger adults also committed less false alarms than children. (E): Working memory score in a auditory digit-sorting task, reflecting the maximum number of digits that were successfully retained and ordered by each participant. Younger adult participants had on average higher working memory capacity compared to children. The reduced number of participants due to data loss caused by technical errors in the WM task. (F): Average congruency effect (RT neutral - RT congruent, in ms) in the Stroop task, separately for both age groups and experiments. Younger adults showed smaller congruency effects. Each dot represents one participant, the black lines indicate boxplots. Bars represent standard error of the mean.

trials $\left(11.5 \%\right.$ vs. $1.4 \%, \chi^{2}(1)=13.1, p<.001$, Fig $\left.2 \mathrm{D}\right)$.

Our control tasks also indicated significant age differences. The verbal working memory test showed that children had a lower working memory span than younger adults (6.3 vs. 10.7 correct answers, respectively, $t(30.9)=-4.3, p<.001)$, see Fig. 2E. Participants also performed a Stroop test in which they needed to respond to the ink color of a written color name (e.g., 'YELLOW' in yellow or red ink) or neutral word ('XXX', colored letters) 
by pressing a button. In children, RTs tended to be slower in trials with the neutral word compared to congruent trials (where color and word agreed), although not significantly, mean difference $25 \mathrm{~ms}, t(21)=1.9, p=.075$. This was not true in young adults, mean difference $-10 \mathrm{~ms}, t(19)=-0.92, p=.369$. Importantly, children had greater RT effects than adults, $t(39.6)=2.0, p=.049$, Fig $2 \mathrm{~F}^{1}$.

\section{Spontaneous strategy discovery and switch}

We next investigated participants' ability to discover and use the alternative strategy. We first assessed to what extent responses in ambiguous trials were based on stimulus color. For instance, if green was paired with left responses in standard trials, we measured the proportion of left responses in spatially ambiguous green trials and vice versa. A mixed effects model revealed an increase in color-based responding over time, i.e. a main effect of Block, $\chi^{2}(1)=4.05, p=.04$, see Fig 3A. There was no evidence that children and adults differed in the extent of color use, i.e. no main effect of age group was found, $\chi^{2}(1)=2.6$, $p=.10$. Pairwise t-tests showed no group differences during any of the blocks. Crucially, testing only behavior in the last 2 blocks before color instructions (i.e., mean of blocks 7 and 8), showed no difference between age groups, with average proportion of color based responding at $58.0 \%$ vs $63.7 \%$ in children and young adults, $\chi^{2}(1)=1.8, p=.28$, see Fig. 3C. Moreover, the proportion of participants who exhibited statistical evidence for color use in the last two correlated blocks (i.e. exhibiting a significant binomial test against 50\%) was $31.8 \%$ among children (7/22), 33.3\% among young adults (7/21) and not statistically different between age groups, $\chi^{2}(1)=0.01, p=1$, see Fig 3D. This result was not affected by the choice of threshold (both $p \mathrm{~s}>.28$ when a higher threshold of at least $75 \%$ or a lower threshold of at least $50 \%$ color use were employed). Likewise, neither proportion of participants who verbally reported that they had discovered the color strategy did not differ

\footnotetext{
${ }^{1}$ Note that because participants were instructed to respond to the ink color, not respond to the written word, the semantic facilitation score reflects a failure of cognitive control. Surprisingly, we did not find age group differences in semantic interference (neutral - incongruent), which were -30ms and $-39 \mathrm{~ms}$ in children and younger adults, respectively, $p=.65$
} 

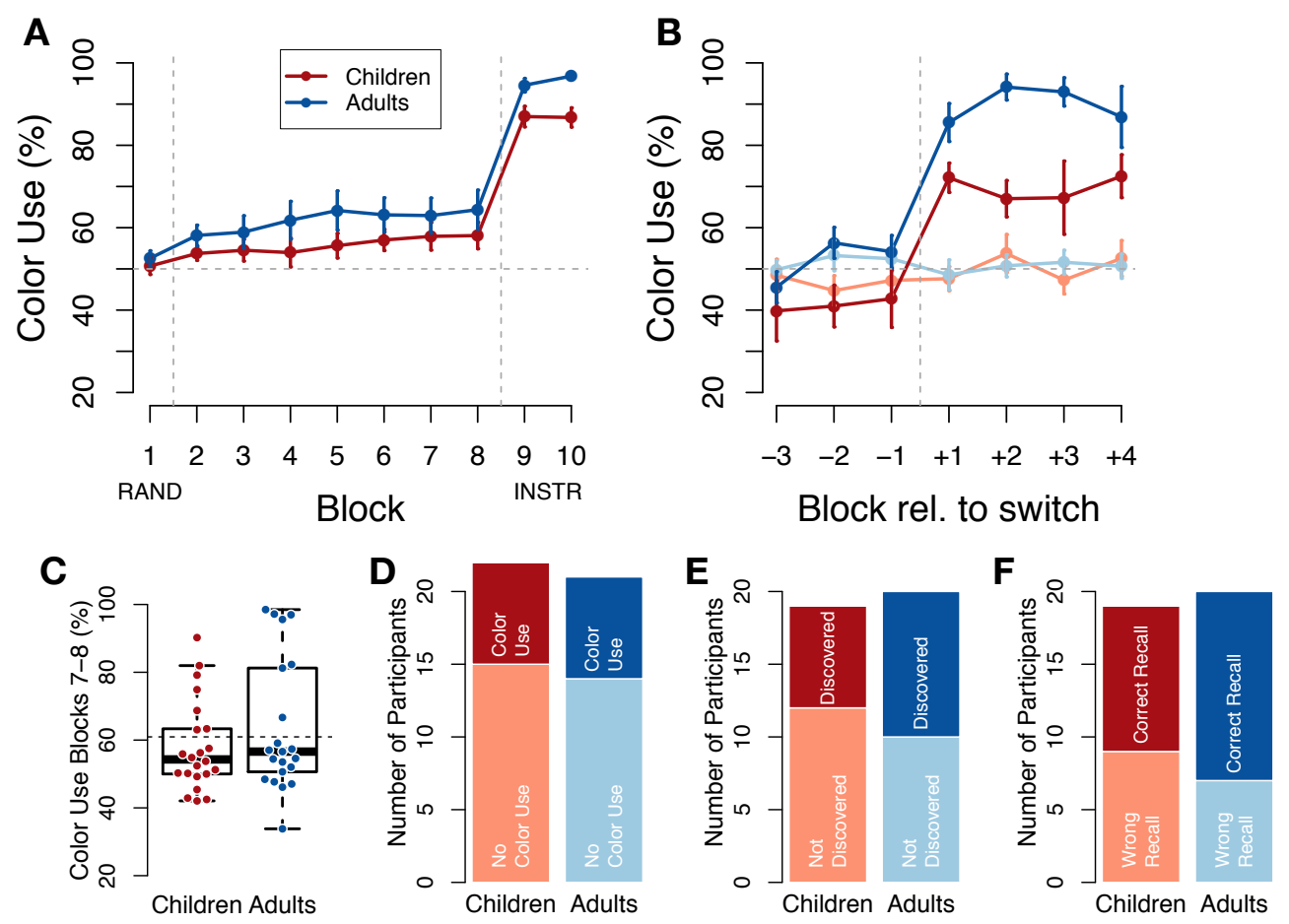

Figure 3: Alternative strategy discovery and use. (A) Percentage of color-based choices ("Color Use") in ambiguous trials as a function of block found in young adults (blue) and children (red). No significant differences were found. (B) Percentage of color use in ambiguous trials time-locked to the mini-block in which a strategy switch was detected. Blocks on the $\mathrm{x}$-axis are split in half relative to A to increase temporal resolution. (C) Percentage of color use in blocks 7 and 8 , before instructions were given. Each dot reflects one participant. (D) Proportion of participants whose behavior indicated a strategy switch towards color based responding by blocks 7 and 8 (>60\% color use). No difference was found between age groups in this measure. (E) Percentage of participants self-reporting discovery of the relation between colors and corners. No age group difference. (F) Percentage of participants able to correctly report the color-corner association after block 8 , but before instructions were given. Different participant numbers in $\mathrm{E} / \mathrm{F}$ vs $\mathrm{D}$ reflect loss of questionnaire data. Bars represent s.e.m.

between age groups ( $p>.05$, Fig. 3E), nor the number of participants who could accurately report the association between color and corners in a questionnaire ( $p>.05$, Fig. 3F).

We next inferred the time-point of discovery from participants' data (see Methods) and time-locked data to this moment, considering only participants with evidence of color use and a time-window ranging from 3 half-blocks before to 3 half-blocks after the switch. This analysis showed that strategy discovery was abrupt, as in our previous study: Pairwise tests in young adults between adjacent blocks showed no evidence of change in color use before or after the switch, while the switch itself was characterized by a clear change, $p=.009$ for 
the comparison -1 to +1 versus $p \mathrm{~s}>.2$ for all other comparisons, corrected, see Fig. 3B. Importantly, the same was true in children, where we also found a significant comparison only for blocks -1 to $+1, p=.008$, but not any other blocks, $p \mathrm{~s}>.8$. This analysis only among participants who switched to color also revealed that younger adults implemented the new strategy with greater accuracy than children. A random effects model including factors of age group and switch (before versus after) revealed a significant interaction, $\chi^{2}(1)=4.4$, $p=0.037$, reflecting that the age groups did not differ before the switch, $p=.27$, but afterwards, $p<.001$ (post-hoc comparisons, adjusted).

\section{Experiment 2}

While Experiment 1 yielded no statistical evidence of any age differences in strategy discovery, it had a limited sample size and was characterized by large age differences in main task performance that led to differences in the number of participants who had to be excluded. In Experiment 2 we therefore repeated Experiment 1 using a slowed-down task version that allowed children to make less mistakes. Our aim was to confirm that no age differences exist even when the performance gap between adults and children is reduced and the number of exclusions equivalent. In addition to such a replication, this approach also offered us the possibility to address power issues by combining data across both experiments.

\section{Methods}

\section{Participants}

Twenty-eight children and 21 young adults were tested in Experiment 2. Following the same exclusion criteria as in Experiment 1, three children and three adults were excluded due to being unable to perform the task during the instructed block. This resulted in an effective sample size of 25 children (10 female) with a mean age of 9.2 years $(\mathrm{SD}=0.8$, range $=8$ to 10 years), and 18 young adults (4 female) with a mean age of 26.6 years $(\mathrm{SD}=4.6$, range $=20$ to 35 years). All participants provided informed consent and the study received ethics 
approval identical to Experiment 1.

\section{Tasks}

Participants performed the same main task as in Experiment 1. To achieve better performance in children, we increased the duration of the stimulus display from $400 \mathrm{~ms}$ to $800 \mathrm{~ms}$. To accommodate for the slower task, participants only received one final instructed block instead of two, reducing the block number from 10 to 9. All other aspects of the main task were identical. The working memory test was identical to Experiment 1. The Stroop task was implemented in psychoPy, but identical otherwise. Stroop data from 2 participants (1 adult) were lost due to technical error.

\section{Procedures}

Procedures were as in Experiment 1.

\section{Analyses}

Analyses followed the same principles as in Experiment 1.

\section{Results}

\section{Performance on standard trials}

Both age groups improved performance in standard trials over the course of blocks 1-8, as reflected in a main effect of Block $\chi^{2}(1)=28.3, p<.001$ (see Fig. 4A). We also found a main effect of Age group, $\chi^{2}(1)=12.9, p<.001$ that was driven by younger adults committing less errors than children (17.6\% vs. 5.5\%; Post-hoc test: $p<.001)$. Differences in error rates between age groups persisted throughout the task, remaining present in the last two blocks before the color instruction (blocks 7-8), $p<.001$. No interaction between Age group and Block was found.

Likewise, RTs on standard trials in Blocks 1 to 8 differed between age groups, with a longer reaction time for children than young adults $\left(\chi^{2}(1)=25.7, p<.001\right)$ and decreased 

${ }_{387} p=.029$.

with practice, $\chi^{2}(1)=21.9, p \mathrm{~s}<.001$ (see Fig. 4B). Age group differences in RT persisted with considerable practice, as shown by planned between-group comparisons of the average RTs in blocks 7 and $8(p<.001)$.

\section{Instructed task execution}

After being provided with instructions to use color in Block 9, adults still outperformed children in terms of accuracy on standard trials. Error rates for adults were significantly lower than in children $(2.7 \%$ vs. $6.9 \%, p<0.01)$. In addition, children benefited more from the instructions than adults, as demonstrated by an interaction between Block ( $7 / 8$ vs. 9) and age group in error rates $\left(\chi^{2}(1)=6.5, p=.011\right)$ and $\mathrm{RTs}\left(\chi^{2}(1)=6.2, p=.013\right)$.

\section{Response inhibition and working memory}

As in Experiment 1, we found age differences in executive control when measured in-task as well as with separate tasks. In-task response inhibition was assessed through false alarm rates in LateGo and NoGo trials. Similar to Experiment 1, children and adults differed markedly in their response inhibition ability, paralleling the performance disparity seen in regular trials. Specifically, compared to young adults, children made significantly more premature key presses (i.e., responses before the frame was displayed, henceforth "False Alarms") in LateGo trials ( $5.6 \%$ vs. $0.7 \%, \chi^{2}(1)=8.9, p=.003$, Fig. 4 C) as well as in NoGo trials $\left(6.1 \%\right.$ vs. $0.9 \%, \chi^{2}(1)=11.6, p<.001$, Fig. $\left.4 \mathrm{D}\right)$.

Young adults also outperformed children on our additional cognitive control tasks. Children had a lower working memory span on the digit-sorting task compared to adults (4.6 vs. 8.7 correct answers, respectively, $t(33.2)=-4.9, p<.001$; see Fig. $4 \mathrm{E})$. On the Stroop task, children showed a larger facilitation effect of congruent stimuli than adults, $t(37.6)=2.3$, 

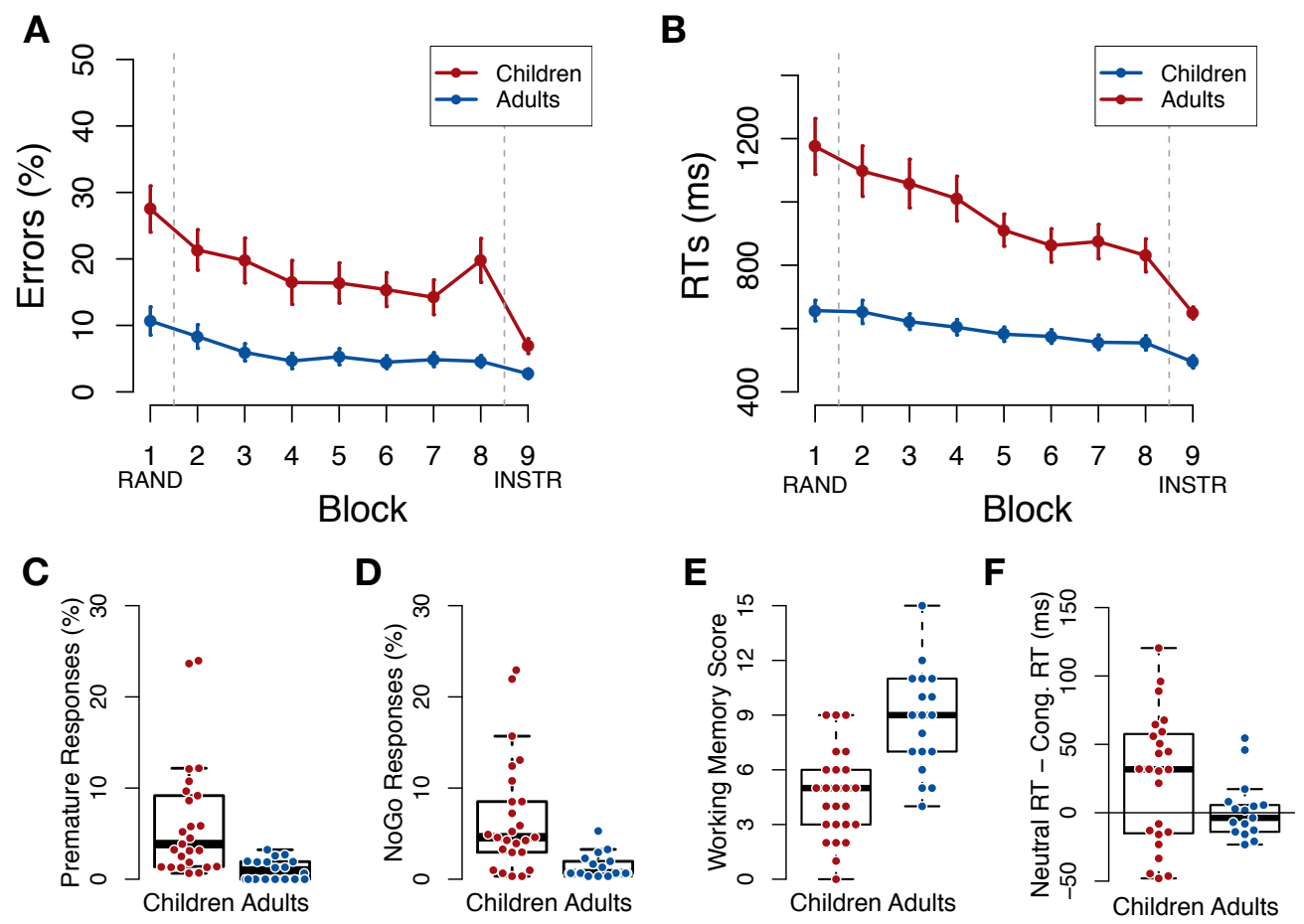

Figure 4: Performance on standard trials and control tasks in Experiment 2. (A): Error rates as a function of block separately for children (red) and younger adults (blue). Age differences in error rates persisted throughout all blocks. (B): Average reaction times (RTs, in milliseconds) over blocks, also indicating sizable and persistent differences between children and young adults. Colors as in panel A. (C): Percentage of false alarms in LateGo trials among young adults (blue) and children (red) in Experiment 2, indicating significantly less errors among young adults. (D): Percentage of false alarms on NoGo trials. As in panel (C), younger adults also committed less false alarms than children. (E): Working memory score in an auditory digit-sorting task, reflecting the maximum number of items that were successfully retained and sorted by each participant. Younger adult participants had on average higher working memory capacity compared to children. (F): Average congruency effect (RT neutral - RT congruent, in ms) in the Stroop task, separately for both age groups. Children showed larger congruency effects, and more variability, than adults. Each dot represents one participant, the black lines indicate boxplots. Bars represent standard error of the mean.

\section{Spontaneous strategy discovery and switch}

We next investigated participants' ability to discover and use the alternative strategy. We first examined the extent of alternative strategy use, defined identically to Experiment 1 as the proportion of color-based decisions on spatially ambiguous trials. A mixed-effects model revealed that color-based decisions increased over time (i.e., a main effect of Block, $\chi^{2}(1)=16.7, p<.001$ ), but did not differ between children and adults (i.e., no main effect of Age group, $\left.\chi^{2}(1)=0.09, p=.76\right)$, see Fig. 5 A. 
Color-based responding on ambiguous trials in the last two correlated blocks, before participants were given explicit instructions to use color, were also examined. There was no difference in the mean proportion of color-based responses between age groups, with proportions of $58.9 \%$ vs $57.4 \%$ in children and young adults respectively, $\chi^{2}(1)=0.12$, $p=.73$, see Fig. 5C. Further, applying the same binomial test criteria as was done in Experiment 1, there were no differences between age groups in the proportion of participants who exhibited statistical evidence for above-chance color use in the last two correlated blocks $\left(\chi^{2}(1)=0.49, p=.528\right)$, with $32 \%(8 / 25)$ of children and $25 \%(4 / 16)$ of adults meeting this criteria (Figure 5D). There remained no significant difference between groups when the threshold for color use was increased to $75 \%, \chi^{2}(1)=0.61, p=.675$, nor decreased to $50 \%$, $\chi^{2}(1)=0.32, p=.752$.

Using the same method as in Experiment 1, we examined the time-points at which participants discovered the alternative color strategy. Among those participants who switched to the alternative strategy, adults and children did not differ in when they discovered the strategy $(p=.932)$.

Unlike in Experiment 1, the age groups also did not differ in the extent to which they implemented the strategy before $(p=.68)$ nor after $(p=0.97)$ discovering the alternative strategy (see Fig. 5B), as assessed through the proportion of color-based responses on ambiguous trials; that is, there was no evidence that adults employed the new strategy with greater efficiency than children.

The proportion of participants who self-reported to have discovered the color strategy (Fig $5 \mathrm{E}$ ) did not differ between age groups (both $p \mathrm{~s}>.05$ ), nor the number of participants who accurately reported the associations between corners and colors in a questionnaire $(p>.05$, Fig $5 \mathrm{~F})$. 

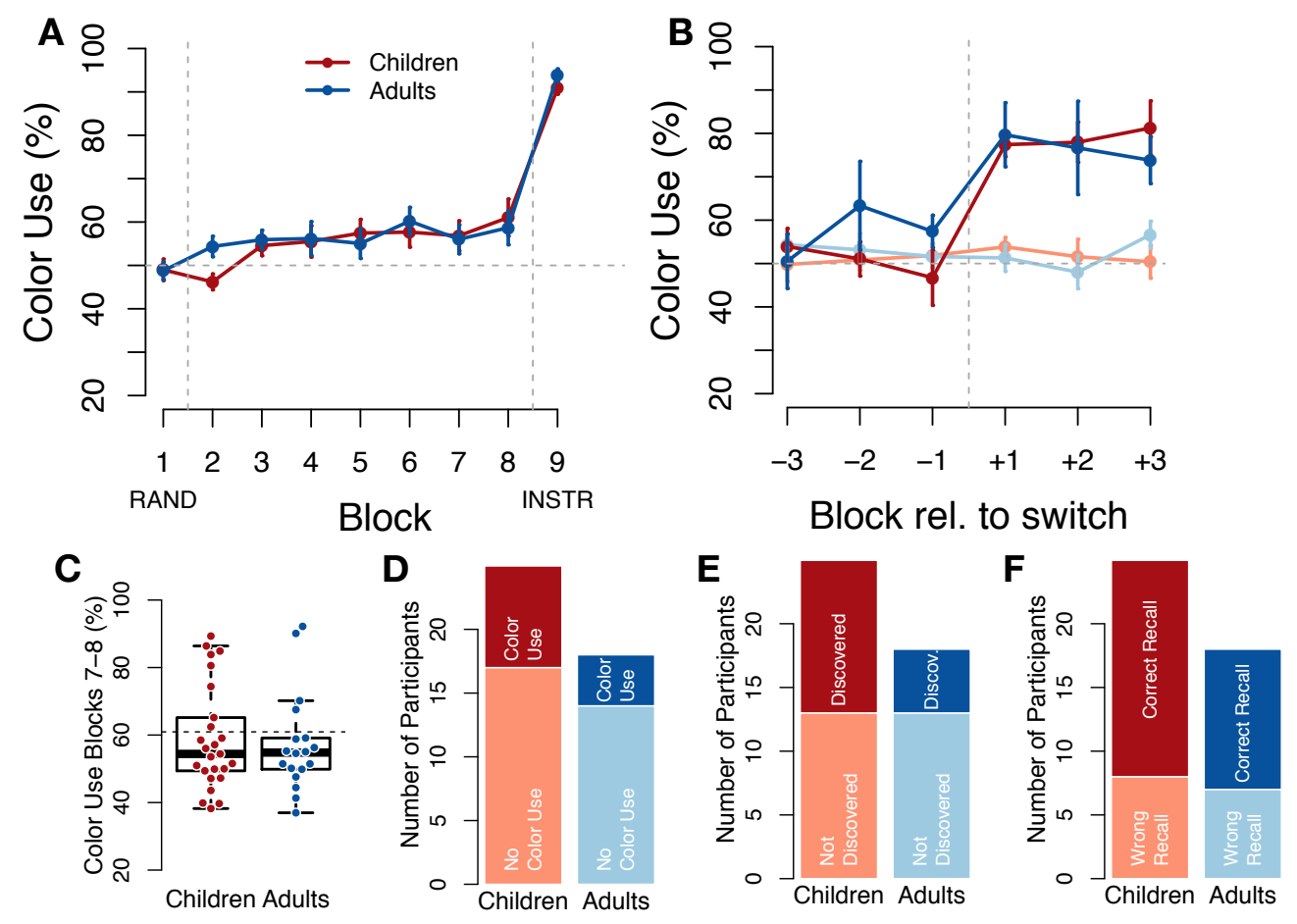

Figure 5: Alternative strategy discovery and use in Experiment 2. (A) Percentage of color-based choices ("Color Use") in ambiguous trials as a function of block found in young adults (blue) and children (red). No significant differences were found. (B) Percentage of color use in ambiguous trials time-locked to the mini-block in which a strategy switch was detected. Blocks on the $\mathrm{x}$-axis are split in half relative to A to increase temporal resolution. (C) Percentage of color use in blocks 7 and 8 , before instructions were given. Each dot reflects one participant. (D) Proportion of participants whose behavior indicated a strategy switch towards color-based responding by blocks 7 and 8 ( $>60 \%$ color use). No difference was found between age groups in this measure. (E) Percentage of participants self-reporting discovery of the relation between colors and corners; no age group difference was found. (F) Percentage of participants able to correctly report the color-corner association after block 8 , but before instructions were given. Bars represent s.e.m.

\section{Combined Analysis}

\section{Age-differences in strategy updating versus age-differences in cognitive control and task performance}

Experiments 1 and 2 yielded no evidence for differences in strategy adaptation between young adults and children, despite the fact that these groups differed substantially in task performance and cognitive control. To reduce the possibility that the lack of evidence could be related to a lack of power, we combined data across both experiments. This resulted in a sample size of 86 participants, consisting of 47 children and 39 young adults. 
An analysis of this combined sample confirmed, unsurprisingly, the considerable agedifferences in task performance, even at the beginning of the task (blocks 1-2): Error rates in regular trials differed between age groups (27.4\% vs. 10.4\%, main effect age group: $\left.\chi^{2}(1)=39.3, p<.001\right)$, and the same main effect of age was found for reaction times $\left(\chi^{2}(1)=48.4, p<.001\right)$. Similarly, premature responses in LateGo and NoGo trials differed significantly across age groups early on in the task $\left(\chi^{2}(1)=11.1\right.$ and 18.4, both $\left.p \mathrm{~s}<.001\right)$ and age differences in the working memory and Stroop tasks were also confirmed in the combined sample (both $p$ s $<.005$, see Fig $6 \mathrm{~A}$, left). Importantly, the same analysis did not find any evidence for differences in strategy updating. The average percentage of color use in blocks $7-8$ was $58.5 \%$ in children and $60.7 \%$ in young adults, not differing significantly between age groups $\left(\chi^{2}(1)=0.4, p=.51\right)$. Among children, $27.5 \%$ of participants (13 out of 47) showed significant evidence for color use by block 7/8. Among adults, $28.2 \%(11 / 39)$ showed evidence for color use, which again provided no evidence that children and adults differ $\left(\chi^{2}(1)<0.1\right)$. In post experimental questionnaires, $43 \%$ of children and $39 \%$ of adults reported to have discovered the color strategy (age differences: $p=.9$ ). Forty-one percent of children versus $24 \%$ of adults reported to have used the strategy $(p=.15)$ and $61 \%$ vs. $63 \%$ correctly reported which color as was associated with which corner $(p>.99)$. Figure $6 \mathrm{~A}$ illustrates the standardized z-scores for each age group across all measures mentioned above.

The sizable differences in task performance and cognitive control therefore stand in contrast to the lack of differences in strategy adaptation. To formally test this impression, we performed a linear mixed effects model in which the z-scored performance in each measure was treated as the dependent variable, and factors Agegroup and Cognitive Ability (cognitive control/task performance versus strategy updating, see Fig 6A.) were tested. As expected, this model indicated a clear interaction of Agegroup and the type of cognitive ability, $\chi^{2}(1)=15.1, p<.001$.

We also tested whether the lack of age differences in the proportion of color use in ambiguous trials could be explained by low reliability in our measures of strategy discovery. 
The split half correlation between color use in odd $(2,4,6,8)$ and even $(3,5,7)$ blocks was $r=.84$ (despite the fact that color use changed across time for some people, as we show in this paper). Constraining the analysis only to periods after the strategy was implemented yielded a correlation of $r=.86$. Hence, our measures of strategy adaptation appear highly reliable.

\section{Relations between strategy discovery, task performance, working memory, and Stroop performance}

Finally, we investigated whether measures of cognitive control and task performance were related to the use of the alternative strategy. We used a linear regression model to predict the logit transformed proportion of color use in ambiguous trials in blocks 7 and 8, using the indicators of cognitive functioning discussed above. Because the measures for standard trial performance (RTs and errors) and response inhibition (premature responses in LateGo and NoGo trials) were highly colinear $(r=.35$ and $r=.89$, respectively), we z-scored and then averaged the affected pairs of variables into singular factors (e.g. on-task performance: z-scored RT + z-scored Error rate). Hence the model included five factors in total: age group, on-task performance, on-task response inhibition, Stroop semantic facilitation effect, and working memory capacity. All main effects were included as well as all pairwise interactions between age group and each of the performance measures. A baseline model that included only age group as a predictor did not indicate any main effect of age group $(p>.05)$ and had significantly worse fit than the full model $\left(r^{2}=.27\right.$ vs. $r^{2}=.001$, Akaike Information Criterion, AIC: 198 vs 207, $p<.001$ ), see Fig. 6B. A stepwise model selection procedure based on AIC confirmed that only the WM main effect and the WM interaction with age group could be removed from the model without loss of fit (final model AIC: 196). Importantly, the full model indicated significant interaction effects of age group and task performance $(p=.02)$ as well as age group and stroop semantic facilitation $(p<.001)$ on the amount of color use. These interactions reflected that the relationship between strategy updating and the other cognitive abilities was negative among young adults, but positive 

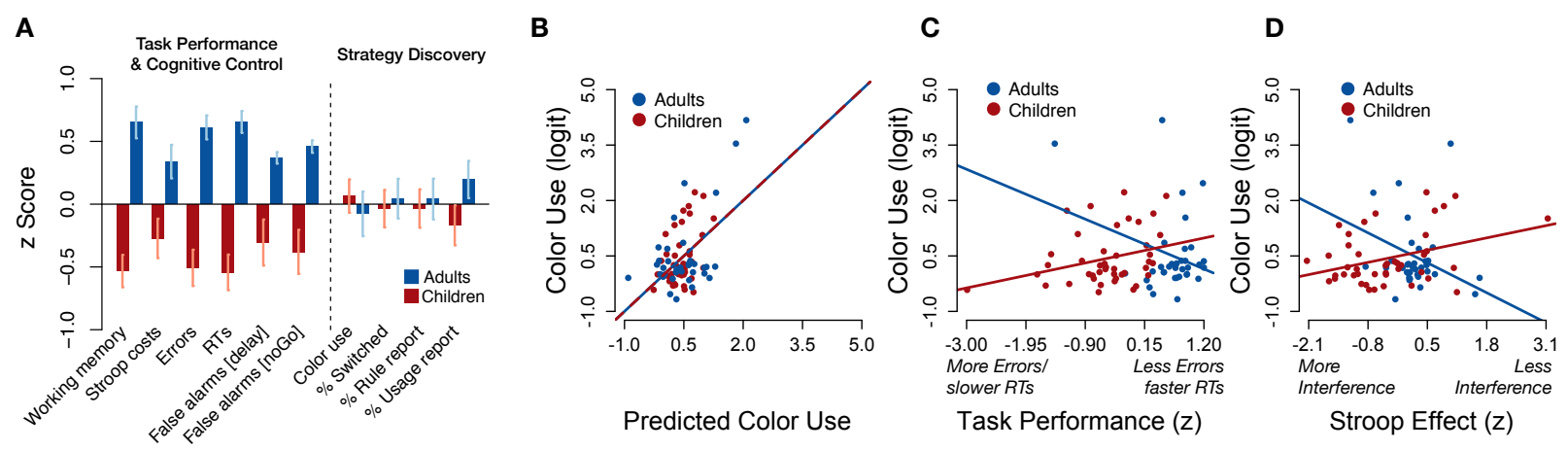

Figure 6: Joint analyses across Experiments 1 and 2 (A) A standardized effect size (z score) was individually calculated for each performance metric for purposes of comparison. Shown metrics reflect data reported in the manuscript in Figures $2-5$. Data collapsed across Experiments 1 and 2. Effects are flipped such that bars higher than 0 indicate that performance for the respective measure was better in one group, i.e. fewer errors, better working memory or faster RTs all are coded as positive values). Data from children is shown in red, and data from adults in blue (as in Fig.s 2-5). Bars represent s.e.m. (B) A linear regression successfully related the logit transformed proportion of color use in Blocks 7 and 8 to the performance factors shown in panel $\mathrm{A}, R^{2}=.27$. (C) The regression model indicated several interactions between age group and cognitive performance. The scatterplot shows task performance (better performance from left to right) is related to logit transformed color use. (D) The scatterplot shows that Stroop semantic facilitation effect (better performance, i.e. less RT costs, from left to right) also had reversed association with color use in young adults versus children. Each dot represents one participant. Lines reflect regression slopes of simple models including only the illustrated factors.

among children. Specifically, better task performance (less errors/faster RTs) was associated with less strategy adaptation in young adults, but the reverse was true among children (Fig $6 \mathrm{C}, r=-.29$ vs. $r=.30$, simple correlations are reported to illustrate the effect). Similarly, less Stroop RT costs were associated with less color use among young adults, $r=-.32$, while the opposite was true among children, $r=.35$, see Fig. $6 \mathrm{D}$.

\section{Discussion}

In two experiments we compared children's and adults' ability to discover possible strategy improvements during task execution. A strategy adaptation occurred when participants changed how they selected their responses throughout the task although a viable response rule was provided at the beginning of the experiment. The instructed task rule allowed error-free task execution, no error feedback was given, and the possibility that an alternative strategy could be found was not mentioned by the experimenters. Strategy improvements 
were therefore a product of participant's self driven exploration of alternative stimulusresponse rules.

Our results showed that strategy adaptations occurred equally often in children and adults. This finding contrasted with the superior performance of adults in all other cognitive abilities that were measured in the same sample, in particular in task execution, working memory, and cognitive control abilities. Flexible strategy updating therefore presents a remarkable exception to the well documented protracted development of decision-making relevant functions in children such as cognitive control (Diamond, 2013; Gur et al., 2012), rule following (Bunge \& Zelazo, 2006), model based decision making (Decker et al., 2016) and choice exploration strategies (Somerville et al., 2017).

Our previous work has shown that strategy updating in our paradigm is a seemingly difficult and rare event even among adults. Specifically, we have found that although the deterministic color-location correlation could be observed in over 700 trials, only about 30\% of young adult participants discovered and used the alternative strategy (Schuck et al., 2015). The present experiment involved around 600 standard trials in which the deterministic color-response relation could be observed. Only 24 out of all 86 tested participants showed behavior consistent with a strategy shift. Eleven of these cases were from the group of 39 younger adults (28\%), and 13 were from the group of 47 tested children (27.5\%). Therefore, our finding of no age difference in strategy updating may seem to stand in contrast to some studies that report better performance of children than younger adults in processing or memory of task-irrelevant information (e.g. Plebanek \& Sloutsky, 2017; Blanco \& Sloutsky, 2019). Differences in task setup may contribute to when no age difference or benefit in children can be expected. Nevertheless, the converging notion is that those mechanisms that protect ongoing task execution may at the same time be detrimental for one's ability to discover the alternative strategy, since the latter involves processing information that is considered irrelevant when following the instructed strategy (Dreisbach \& Fröber, 2018, Goschke, 2000). One additionally relevant idea in this regard is the notion of developmental changes in Bayesian learning, which suggests that weaker priors in children lead to larger 
updates of their posterior in light of the same observation, i.e. a stronger consideration of alternative hypotheses that are consistent with new evidence (e.g. Gopnik \& Wellman, 2012, Xu \& Kushnir, 2013). Studies of causal reasoning indeed showed that children, preschoolers particularly, are more flexible than older children and adults in adopting unfamiliar hypotheses that are consistent with new evidence (Gopnik et al., 2017, Lucas, Bridgers, Griffiths, \& Gopnik, 2014). While our data does not suggest an age-related difference favoring children, the Bayesian framework may be an useful general approach that captures the contextual factors that affect how strongly the instructed strategy is being executed as prior belief. In combination, these factors could explain why children who are almost four times as error prone as adults in executing the instructed strategy $(20.6 \%$ vs. $5.5 \%$ by the end of the experiment, blocks 7-8) are equally good in the complex ability to adapt their ongoing decision making strategy.

Notably, we found different associations between the amount of color use (strategy adaptation) and performance in other tasks among children versus adults. Most interestingly, the Stroop (semantic facilitation) effect correlated positively with color use among children $(r=.35$, whereby larger Stroop effects indicate less interference, see Fig. 6D). This hints at a 'beneficial' effect of more cognitive control. In young adults, in contrast, we found a negative relation between the Stroop effect and color use , $r=-.32$, indicating that young adults with better executive functions were less influenced by the color, see Fig 6D. The same pattern of results was found when investigating task performance. While the present study was not designed to specifically examine the question about the factors influencing strategy discovery, the available data thus could hint at two possible explanations. On the one hand, relatively better task and Stroop performance could reflect different mechanisms in adults versus children. Whereas in adults good performance mainly reflects attentional focus that is detrimental to strategy updating, in children relatively good task performance could reflect different factors, such as better encoding of the instructions or better motor control. On the other hand, given the overall performance difference between children and adults it also seems possible that the opposite-sign relations to strategy switching reflect 
an inverted U-shaped relationship between attentional mechanisms and strategy switching. Further investigations are therefore needed that shed light on the factors that facilitate and impede strategy discovery, for instance testing Bayesian learning abilities more directly and utilizing update focused working memory measures such as n-back or AX-CPT tasks. In addition, given the between subject nature of the effects, larger sample sizes that yield higher power for detecting small difference between age groups will be needed.

Which computational properties allow a decision-maker to find new solutions within previously ignored environmental structure remains an overall unsolved problem that relates to the general question of representation learning mechanisms in the brain (e.g. Niv, 2019 Schuck, Wilson, \& Niv, 2018), a topic that has also been considered in developmental psychology (e.g. Karmiloff-Smith, 1992). It also remains unclear how the high levels of flexible updating could be neurally implemented in the still developing brain. Our own investigation in younger adults suggested that the spontaneous change in strategy relied on a internal simulation mechanism in medial prefrontal cortex (mPFC). In children, mPFC displays a complex structural maturation trajectory that differs between its subregions (Shaw et al., 2008), with the orbital parts following an early maturation pattern, whereas the dorsal parts follow a late maturation pattern. The cluster of mPFC found in Schuck et al. (2015) corresponds to the region that goes through structural transition between 8 and 10 years of age. In addition, it remains unclear whether children's brains exhibit similar dynamics in long range brain activity correlations that have been associated with our task (Allegra et al., 2020), given the marked changes in brain network segregation observed in children (Baum et al., 2017). This may be relevant insofar as prefrontal network dynamics have been linked to the balance between cognitive stability and flexibility (e.g., Durstewitz, Seamans, \& Sejnowski, 2000), suggesting that the stable states that correspond to task sets representations can be thought of as basins in a potential landscape of network state. According to this view, deeper basins are related to cognitive stability and efficient task execution, while shallower basins imply less effort to switch but higher susceptibility to distraction. In line with this idea it has been found that depth of the attractor state, as indexed by functional coupling 
between prefrontal areas, is related to how readily individuals switch from one task state to another in the light of ambiguous task cues (Armbruster, Ueltzhoeffer, Basten, \& Fiebach, 2012). Therefore, the development of attractor stability of prefrontal networks may be a useful topic for future investigations (see also, Baum et al., 2017).

In summary, the present study has shown that the ability to perform strategy adaptations presents a remarkable exception from children's comparatively limited decision making skills, such as executing simple task rules, holding information in working memory and inhibiting prepotent responses. The comparatively well developed ability to discover novel strategies for a known task in children might offer a unique opportunity for educators in fostering learning in children. More generally, our findings highlight that the development of cognitive functions in children might result in complex dynamics of abilities that rely on the interaction of several cognitive functions.

\section{Data Availability}

The legal possibilities of making anonymized raw data openly available given the used consent forms are evaluated at the moment. Contingent on approval by the local data protection office and the ethical review board, all raw and aggregate data used to generate results in Figures 2-5 will be made publicly available upon publication.

\section{Code Availability}

All code used to generate results in Figures 2-5 will be made publicly available upon publication.

\section{Author Contributions}

NWS, RG, DW and YLS designed research. DSA and AL conducted research. NWS, YLS and AXL drafted the manuscript. NWS, AXL and YLS analyzed data. All authors contributed to interpreting the data and writing the manuscript. All authors gave final approval for publication and agree to be held accountable for the work performed therein. 


\section{Acknowledgments}

NWS was funded by an Independent Max Planck Research Group grant awarded by the Max Planck Society and a Starting Grant from the European Union (ERC-2019-StG-REPLAY852669). DW was funded by DFG grant WE2852/3-1. YLS was funded by a Minerva Research Group by the Max Planck Society, a Starting Grant from the European Union (ERC-2018-StG-PIVOTAL-758898), and a Fellowship from the Jacobs Foundation (JRF 2018-2020).

\section{References}

Allegra, M., Seyed-Allaei, S., Schuck, N. W., Amati, D., Laio, A., \& Reverberi, C. (2020). Brain network dynamics during spontaneous strategy shifts and incremental task optimization. NeuroImage, 217, 116854. doi:10.1016/j.neuroimage.2020.116854

Armbruster, D. J. N., Ueltzhoeffer, K., Basten, U., \& Fiebach, C. J. (2012). Prefrontal cortical mechanisms underlying individual differences in cognitive flexibility and stability. Journal of Cognitive Neuroscience, 24(12), 2385-2399. doi: $10.1162 /$ jocn $\left\{\backslash_{-}\right\} \mathrm{a}\left\{\backslash_{-}\right\} 00286$

Bates, D., Mächler, M., Bolker, B., \& Walker, S. (2015). Fitting Linear Mixed-Effects Models Using lme4. Journal of Statistical Software, 67(1), 51. doi:10.18637/jss.v067.i01

Baum, G. L., Ciric, R., Roalf, D. R., Betzel, R. F., Moore, T. M., Shinohara, R. T., ... Satterthwaite, T. D. (2017). Modular Segregation of Structural Brain Networks Supports the Development of Executive Function in Youth. Current Biology, 27(11), 1561-1572. doi:10.1016/j.cub.2017.04.051

Blakemore, S. J. \& Choudhury, S. (2006). Development of the adolescent brain: Implications for executive function and social cognition. Journal of Child Psychology and Psychiatry and Allied Disciplines, 47(3-4), 296-312. doi:10.1111/j.1469-7610.2006.01611.x 
Blanco, N. J. \& Sloutsky, V. M. (2019). Adaptive Flexibility in Category Learning? Young Children Exhibit Smaller Costs of Selective Attention Than Adults. Developmental Psychology. doi:10.1037/dev0000777

Bonawitz, E., Denison, S., Griffiths, T. L., \& Gopnik, A. (2014). Probabilistic models, learning algorithms, and response variability: Sampling in cognitive development. doi:10.1016/j.tics.2014.06.006

Bunge, S. A. \& Wright, S. B. (2007). Neurodevelopmental changes in working memory and cognitive control. Current Opinion in Neurobiology, 17(2), 243-250. doi: $10.1016 /$ j.conb.2007.02.005

Bunge, S. A. \& Zelazo, P. D. (2006). A brain-based account of the development of rule use in childhood. Current Directions in Psychological Science, 15(3), 118-121. doi:10.1111/j.0963-7214.2006.00419.x

Corbetta, M. \& Shulman, G. L. (2002). Control of goal-directed and stimulus-driven attention in the brain. Nature Reviews Neuroscience, 3(3), 201-215. doi: $10.1038 / \operatorname{nrn} 755$

Davidson, M. C., Amso, D., Anderson, L. C., \& Diamond, A. (2006). Development of cognitive control and executive functions from 4 to 13 years: Evidence from manipulations of memory, inhibition, and task switching. Neuropsychologia, 44(11), 2037-2078. doi:10.1016/j.neuropsychologia.2006.02.006

Decker, J. H., Lourenco, F. S., Doll, B. B., \& Hartley, C. a. (2015). Experiential reward learning outweighs instruction prior to adulthood. Cognitive, Affective, $\&$ Behavioral Neuroscience. doi:10.3758/s13415-014-0332-5

Decker, J. H., Otto, A. R., Daw, N. D., \& Hartley, C. A. (2016). From Creatures of Habit to Goal-Directed Learners: Tracking the Developmental Emergence of Model-Based Reinforcement Learning. Psychological Science, $27(6), 848-858$. doi: $10.1177 / 0956797616639301$

Demetriou, A., Christou, C., Spanoudis, G., \& Platsidou, M. (2002). The development of mental processing: efficiency, working memory, and thinking. Monographs of the 
Society for Research in Child Development, 67(1), 1-154. doi: $10.1111 / 1540-5834.671173$

Diamond, A. (2013). Executive Functions. Annual Review of Psychology, 64(1), 135-168. doi:10.1146/annurev-psych-113011-143750

Dreisbach, G. \& Fröber, K. (2018). On How to Be Flexible (or Not): Modulation of the Stability-Flexibility Balance. Current Directions in Psychological Science, Online First. doi: $10.1177 / 0963721418800030$

Dreisbach, G. \& Haider, H. (2008). That's what task sets are for: shielding against irrelevant information. Psychological research, 72(4), 355-61. doi:10.1007/s00426-007-0131-5

Dreisbach, G. \& Wenke, D. (2011). The shielding function of task sets and its relaxation during task switching. doi:10.1037/a0024077

Durstewitz, D., Seamans, J. K., \& Sejnowski, T. J. (2000). Dopamine-Mediated Stabilization of Delay-Period Activity in a Network Model of Prefrontal Cortex. Journal of Neurophysiology, 83(3), 1733-1750. doi:10.1152/jn.2000.83.3.1733

Evans, J. L., Saffran, J. R., \& Robe-Torres, K. (2009). Statistical Learning in Children With Specific Language Impairment. Journal of Speech Language and Hearing Research, 52(2), 321. doi:10.1044/1092-4388(2009/07-0189)

Feo, V. D., Panzeri, S., \& Dehaene, S. (2018). Learning to focus on number. Cognition, 181 (June 2017), 35-45. doi:10.1016/j.cognition.2018.07.011

Fischer, B., Biscaldi, M., \& Gezeck, S. (1997). On the development of voluntary and reflexive components in human saccade generation. Brain Research, 754 (1-2), 285-297. doi:10.1016/S0006-8993(97)00094-2

Fukushima, J., Hatta, T., \& Fukushima, K. (2000). Development of voluntary control of saccadic eye movements: I. Age- related changes in normal children. Brain and Development, 22(3), 173-180. doi:10.1016/S0387-7604(00)00101-7 
Garon, N., Bryson, S. E., \& Smith, I. M. (2008). Executive Function in Preschoolers: A Review Using an Integrative Framework. Psychological Bulletin, 134(1), 31-60. doi:10.1037/0033-2909.134.1.31

Gaschler, R., Schuck, N. W., Reverberi, C., Frensch, P. A., \& Wenke, D. (2019). Incidental Covariation Learning Leading to Strategy Change. PloS one, in press.

Gaschler, R., Vaterrodt, B., Frensch, P. A., Eichler, A., \& Haider, H. (2013). Spontaneous Usage of Different Shortcuts Based on the Commutativity Principle. PLoS ONE, 8(9), 1-13. doi:10.1371/journal.pone.0074972

Gopnik, A., O’Grady, S., Lucas, C. G., Griffiths, T. L., Wente, A., Bridgers, S., ... Dahl, R. E. (2017). Changes in cognitive flexibility and hypothesis search across human life history from childhood to adolescence to adulthood. Proceedings of the National Academy of Sciences, 114(30), 7892-7899. doi:10.1073/pnas.1700811114

Gopnik, A. \& Wellman, H. M. (2012). Reconstructing constructivism: Causal models, Bayesian learning mechanisms, and the theory theory. Psychological Bulletin, 138(6), 1085-1108. doi:10.1037/a0028044

Goschke, T. (2000). Intentional Reconfiguration and Involuntary Persistence in Task Set Switching. In Control of cognitive processes: Attention and performance xviii (p. 331).

Gur, R. C., Richard, J., Calkins, M. E., Chiavacci, R., Hansen, J. A., Bilker, W. B., ... Gur, R. E. (2012). Age group and sex differences in performance on a computerized neurocognitive battery in children age 8-21. Neuropsychology, 26(2), 251-265. doi: $10.1037 / \mathrm{a} 0026712$

Hartley, C. A. \& Somerville, L. H. (2015). The neuroscience of adolescent decision-making. Current Opinion in Behavioral Sciences, 5, 108-115. doi:10.1016/j.cobeha.2015.09.004 Karmiloff-Smith, A. (1992). Beyond Modularity: A Developmental Perspective on Cognitive Science. Cambridge, MA: MIT Press. doi:10.3109/13682829409041485

Kharitonova, M., Martin, R. E., Gabrieli, J. D., \& Sheridan, M. A. (2013). Cortical gray-matter thinning is associated with age-related improvements on executive 
function tasks. Developmental Cognitive Neuroscience, 6(6), 61-71.

doi:10.1016/j.den.2013.07.002

Lieder, F. \& Griffiths, T. L. (2017). Strategy selection as rational metareasoning. Psychological Review, 124(6), 762-794. doi:10.1037/rev0000075

Lucas, C. G., Bridgers, S., Griffiths, T. L., \& Gopnik, A. (2014). When children are better (or at least more open-minded) learners than adults: Developmental differences in learning the forms of causal relationships. Cognition, 131(2), 284-299. doi:10.1016/j.cognition.2013.12.010

Luna, B., Padmanabhan, A., \& O'Hearn, K. (2010). What has fMRI told us about the Development of Cognitive Control through Adolescence? Brain and Cognition, 72(1), 101-113. doi:10.1016/j.bandc.2009.08.005

Marewski, J. N. \& Link, D. (2014). Strategy selection: An introduction to the modeling challenge. Wiley Interdisciplinary Reviews: Cognitive Science, 5(1), 39-59. doi: $10.1002 /$ wcs. 1265

Niv, Y. (2019). Learning task-state representations. Nature Neuroscience, 22(10), 1544-1553. doi:10.1038/s41593-019-0470-8

Plebanek, D. J. \& Sloutsky, V. M. (2017). Costs of Selective Attention: When Children Notice What Adults Miss. Psychological Science, 28(6), 723-732. doi: $10.1177 / 0956797617693005$

R Core Team. (2018). R: A Language and Environment for Statistical Computing. Vienna, Austria: R Foundation for Statistical Computing.

Ridderinkhof, K. R., Van Der Molen, M. W., Band, G. P., \& Bashore, T. R. (1997). Sources of interference from irrelevant information: A developmental study. Journal of Experimental Child Psychology, 65(3), 315-341. doi:10.1006/jecp.1997.2367

Saffran, J. R., Aslin, R. N., \& Newport, E. L. (1996). Statistical Learning by 8-Month-Old Infants. Science, 274(5294), 1926-1928. doi:10.1126/science.274.5294.1926

Sakai, K. (2008). Task set and prefrontal cortex. Annual Review of Neuroscience, 31, 219-245. 
Schuck, N. W., Gaschler, R., Wenke, D., Heinzle, J., Frensch, P. A., Haynes, J.-D., \& Reverberi, C. (2015). Medial Prefrontal Cortex Predicts Internally Driven Strategy Shifts. Neuron, 86(1), 331-340. doi:10.1016/j.neuron.2015.03.015

Schuck, N. W., Wilson, R., \& Niv, Y. (2018). A State Representation for Reinforcement Learning and Decision-Making in the Orbitofrontal Cortex. In Goal-directed decision making (pp. 259-278). Elsevier. doi:10.1016/B978-0-12-812098-9.00012-7

Schulz, E., Wu, C. M., Ruggeri, A., \& Meder, B. (2019). Searching for Rewards Like a Child Means Less Generalization and More Directed Exploration. Psychological Science, 30 (11), 1561-1572. doi:10.1177/0956797619863663

Shaw, P., Kabani, N. J., Lerch, J. P., Eckstrand, K., Lenroot, R., Gogtay, N., ... Wise, S. P. (2008). Neurodevelopmental Trajectories of the Human Cerebral Cortex. Journal of Neuroscience, 28(14), 3586-3594. doi:10.1523/JNEUROSCI.5309-07.2008

Siegler, R. S. (1995). Children's thinking: How does change occur. In Memory performance and competencies: Issues in growth and development (pp. 405-430).

Siegler, R. S. (1996). A grand theory of development. Monographs of the Society for Research in Child Development, 61(1-2), 266-275. doi:10.1111/j.1540-5834.1996.tb00550.x

Siegler, R. S. (1997). Emerging Minds: The Process of Change in Children's Thinking. New York: Oxford University Press.

Siegler, R. S. (2006). Microgenetic analyses of learning. In Handbook of child psychology (pp. 464-510).

Somerville, L. H., Sasse, S. F., Garrad, M. C., Drysdale, A. T., Akar, N. A., Insel, C., \& Wilson, R. C. (2017). Charting the expansion of strategic exploratory behavior during adolescence. Journal of Experimental Psychology: General, 146(2), 155-164. doi: $10.1037 /$ xge0000250

Tipper, S. P., Bourque, T. A., Anderson, S. H., \& Brehaut, J. C. (1989). Mechanisms of attention: A developmental study. Journal of Experimental Child Psychology, 48(3), 353-378. doi:10.1016/0022-0965(89)90047-7 
Xu, F. \& Kushnir, T. (2013). Infants Are Rational Constructivist Learners. Current

Directions in Psychological Science, 22(1), 28-32. doi:10.1177/0963721412469396 